\title{
Optimisation des conditions de régénération de plantes fertiles à partir de suspensions cellulaires issues de cals embryogenèse d'apex caulinaire de mil (Pennisetum glaucum (L.) R.)
}

\author{
Kouakou TIÉCOURA ${ }^{1}$, Oulo ALLA-N'NAN ${ }^{1 *}$, Abou Bakari KOUASSI ${ }^{1}$, \\ Monique DINANT ${ }^{2}$ et Lucien LEDOU $^{2}$ \\ ${ }^{1}$ Laboratoire de Génétique, UFR Biosciences, Université Félix HOUPHOUËT-BOIGNY, 22 BP 582 Abidjan \\ 22, Côte d'Ivoire. \\ ${ }^{2}$ Laboratoire de Génétique Moléculaire, Département de Botanique, Université de Liège, Sart Tilman, B22, B- \\ 4000 Liège, Belgique. \\ *Auteur correspondant, E-mail: nanoulo@yahoo.fr; 22 BP 582 Abidjan 22, Côte d'Ivoire. \\ Tél: 0022507164327
}

\section{RÉSUMÉ}

Ce travail a pour objectif d'établir les conditions optimales de régénération de plantes fertiles à partir de suspensions cellulaires embryogènes en vue de leur utilisation pour une transformation génétique par bombardements de protoplastes. Ce travail réalisé sur des variétés de Pennisetum glaucum de Côte d'Ivoire a permis de mettre en évidence les facteurs qui influent (taille et âge des cals, milieu de conditonnement, et âge de la suspension cellulaire) sur la régénération de plantes fertiles. Il ressort de cette étude que la taille des cals et les milieux de conditionnement utilisés n'ont aucun effet sur le taux de régénération de plantes fertiles. Cependant, l'âge de la suspension cellulaire obtenue à partir de cals embryogènes et l'âge du cal initiateur de la suspension cellulaire influent sur le taux de régénération. Le taux de plantes normales régénérées est élevé durant les deux premiers mois de culture de la suspension cellulaire initiée à partir des cals de 2-3 mois (60 à $70 \%$ ). Les plantes régénérées présentent les mêmes caractéristiques de croissance végétative (taille, longueur de la chandelle, nombre de barres, longueur de l'épi) que les plantes témoins. Pour le rendement, les plantes régénérées portent moins de grains (412) que les plantes témoins (715).

(C) 2014 International Formulae Group. All rights reserved.

Mots clés : Plantes fertiles, suspension cellulaire, cal embryogène, Pennisetum glaucum, Côte d'Ivoire.

\section{INTRODUCTION}

La régénération de plantes à partir de protoplastes dépend de la qualité des suspensions cellulaires embryogènes. $\mathrm{La}$ qualité de la suspension cellulaire s'exprime par sa capacité à régénérer des plantes fertiles. En effet, les suspensions cellulaires embryogènes sont utilisées comme cibles pour la transformation des céréales par bombardement de particules enrobées d'ADN. Wilmar et al. (1968) ont été les premiers à montrer à partir d'hypocotyle d'Asparagus officinalis que la capacité de régénération de plantes à partir des suspensions cellulaires est fonction du temps de culture de celles-ci. Depuis ces travaux, de nombreuses études 
portant sur la régénération de plantes à partir de suspensions cellulaires ont été rapportées chez d'autres monocotylédones. Divers explants ont été utilisés au cours de ces travaux. Il s'agit notamment:

- d'embryons immatures et de jeunes inflorescences de Panicum maximum (Lu et al., 1981; Gnanapragasam et al., 1992) et de Zea mays (Kamo et al., 1986; Shillito et al., 1989).

- d'embryons matures de Hordeum vulgare (Sharma et al., 2005)

- des bases de feuilles, de racines et d'apex caulinaires de Oryza sativa (Zimny et al., 1986; Arockiasamy et Ignacimuthu, 2007; Mohana et al., 2011).

- des graines, des inflorescences et bases de feuilles de Triticum aestivum (Karp et al., 1987; Mejza et al., 1993; Winfield et al., 1993; Haliloglu, 2006).

Chez le maïs, des difficultés de régénération de plantes à partir de suspensions cellulaires ont été évoquées pour certains génotypes (Kamo et al., 1986).

En ce qui concerne le mil, Pennisetum glaucum ou Pennisetum americanum, plusieurs travaux ont permis la régénération de plantes à partir de suspensions cellulaires obtenues avec différents explants (Vasil et Vasil, 1981, 1982; Vasil, 1994; Devi et al., 2000; Oldach et al., 2001; Arockiasamy et al., 2006; Jha et al., 2009). Dans toutes ces études, l'apex caulinaire n'est généralement pas l'explant utilisé et, lorsque c'est le cas (Lambé et al., 1995; 2000), la régénération de plantes à partir des suspensions cellulaires n'a pas fait l'objet d'étude approfondie. Après avoir déterminé les conditions optimales d'établissement de suspensions cellulaires embryogènes (Tiécoura et al., 2014), ce travail, présente pour la première fois, et à partir de la variété NE (Nord Est) de Pennisetum glaucum de Côte d'Ivoire, les facteurs influençant la régénération de plantes fertiles à partir de ces suspensions cellulaires issues de cals d'apex caulinaires.

\section{MATÉRIEL ET MÉTHODES \\ Matériel végétal}

Le matériel végétal est constitué de méristèmes apicaux caulinaires isolés de la variété Nord-Est (Variété NE) de Pennisetum glaucum. Cette variété a été sélectionnée pour ses qualités organoleptiques et agromorphologiques. Elle est cultivée au Nord Est de la Côte d'Ivoire. L'isolement des explants, l'induction de la callogenèse et l'obtention de suspensions cellulaires embryogènes ont été effectués selon les protocoles décrits par Tiécoura et al. (2003). Les suspensions cellulaires embryogènes obtenues ont été utilisées pour la régénération de plantes fertiles.

\section{Les milieux de culture utilisés}

Trois milieux de culture ont été utilisés pour le conditionnement des cals et la régénération de plantes. Ces milieux sont ceux les plus recommandés pour la culture des cals de mil. Il s'agit du milieu MS (1. 1. 2,5) : milieu de Murashige et Skoog (MS) additionné de $1 \mathrm{mg} / \mathrm{l}$ de BAP, de $1 \mathrm{mg} / \mathrm{l} \mathrm{de}$ ANA et de 2,5 mg/l de 2,4-D (Acide 2,4dichlorophénoxyacétique); du milieu MS(5) : Murashige et Skoog (MS) additionné de 5 $\mathrm{mg} / \mathrm{l}$ de2,4-D et du milieu N6(1. 100. 25) : milieu de Chu additionné de $1 \mathrm{mg} / \mathrm{l}$ de $2,4 \mathrm{D}$, $100 \mathrm{mg} / \mathrm{l} \mathrm{d}$ 'hydrolysat de caséine et $25 \mathrm{mg} / \mathrm{l}$ de proline (Tiécoura et al., 2003). Les différentes fractions de cals ont été conditionnées sur ces différents milieux avant leur transfert sur le milieu MS (0,5.2) : milieu Mirashige et Skoog additionné de $0,5 \mathrm{mg} / \mathrm{l}$ ANA et de $2 \mathrm{mg} / \mathrm{l}$ de BAP pour la régénération de plantes fertiles. Pour chaque fraction, 60 cals ont été évalués pour leur capacité à régénérer des plantes.

Évaluation de l'effet de la taille de la fraction de suspension cellulaire sur le taux de régénération de plantes

Des cals embryogènes développés pendant environ 2 mois sur du milieu de 
Murashige et Skoog (1962) additionné de 1 mg/l d'ANA (acide naphtalène acétique), 1 $\mathrm{mg} / \mathrm{l}$ de BAP (benzylaminopurine) et $2,5 \mathrm{mg} / 1$ de 2,4-D (acide 2,4-dichloro phénoxyacétique), [milieu MS (1. 1. 2,5)], ont été utilisés pour initier des suspensions cellulaires dans du milieu $\operatorname{MS}(2)$ : milieu Murashige et Skoog additionné de $2 \mathrm{mg} / \mathrm{l}$ de 2,4 D. Tous les mois, une partie de suspension cellulaire a été utilisée pour la subculture et l'autre partie a été filtrée sur des tamis de 250 ou $630 \mu \mathrm{m}$ de diamètre de maille. Cette filtration permet d'obtenir trois fractions d'amas cellulaires :

1- $\quad$ les cals de dimensions supérieures à $630 \mu \mathrm{m}$;

2- les cals de dimensions comprises entre $250 \mu \mathrm{m}$ et $630 \mu \mathrm{m}$;

3- les cals de dimensions inférieures à $250 \mu \mathrm{m}$.

Des échantillons de chaque fraction ont été cultivés pendant une semaine sur milieux solides de conditionnement MS (1. 1. 2,5) pour les raffermir. Ces cals ont ensuite été placés sur du milieu Murashige et Skoog (1962) additionné de 0,5 ANA et $2 \mathrm{mg} / \mathrm{l}$ de BAP) [milieu MS (0,5.2)] pour la régénération des plantes (Figure 1d). Soixante (60) cals de chaque fraction d'amas cellulaires ont été évalués pour leur capacité à régénérer des plantes.

Évaluation des effets de l'âge du cal initiateur de la suspension cellulaire et de l'âge de la suspension cellulaire sur le taux de régénération de plantes

Des cals embryogènes âgés de 2,6 et 12 mois (Figure 1a), cultivés sur milieu MS (1. 1. 2,5), ont été utilisés pour initier des suspensions cellulaires embryogènes (Figure 1b) dans du milieu MS(2). Après chaque subculture, tous les mois, le reste de la suspension cellulaire a été filtrée avec un tamis de $250 \mu \mathrm{m}$ de diamètre de maille. Seuls les cals de la fraction I (les cals de dimensions inférieures à $250 \mu \mathrm{m}$ ) ont été conditionnés sur le milieu MS (1. 1. 2,5) pour une semaine. Ces cals ont ensuite été transférés sur le milieu MS $(0,5$. 2) pour la régénération de plantes. Soixante (60) cals ont été évalués pour leur capacité à régénérer des plantes. Le choix de la fraction I tient au fait que cette fraction est la mieux indiquée pour les extractions de protoplastes et les transformations génétiques (Tiécoura et al., 2001).

Évaluation des effets de l'âge du cal initiateur de la suspension cellulaire et de l'âge de la suspension cellulaire sur la qualité des plantes régénérées

Les plantes régénérées ont été observées et jugées anormales si elles présentaient des malformations (des feuilles en aiguilles, en rosette ou blanches) (Figure 1c), ou normales si elles n'en présentaient pas (Figure 1d). Des suspensions cellulaires âgés de 1 à 4 mois initiées avec cals de 2 à 12 mois ont été évalués pour leurs effets sur le taux de plantes normales.

Évaluation des effets de l'âge du cal initiateur de la suspension cellulaire et de l'âge de la suspension cellulaire sur le taux des plantes albinos régénérées

Les plantes régénérées ont été observées et jugées albinos si elles présentaient des feuilles blanches. Les cals initiateurs âgés de 2 à 12 mois et des suspensions cellulaires âgés de 1 à 4 mois ont été évalués pour leurs effets sur le taux de plantes albinos.

Comparaison des caractéristiques des plantes régénérées à celles des plantes issues de semis

Des plantes portant 4 à 6 feuilles, régénérées à partir de suspensions cellulaires issues de cals âgés de 2 à 3 mois (30 plantes), ont été mises en culture dans la serre pendant 60 jours avec une photopériode de $16 \mathrm{~h}$, pour le développement végétatif, avant de passer à une photopériode de $12 \mathrm{~h}$ pour la floraison (Tiécoura et al., 2003). La taille de la plante, le nombre de barres, la longueur de la 
chandelle, la longueur de l'épi et le nombre de grains par plante ont été évalués.

\section{Analyse statistique}

Les données ont analysées avec le logiciel STATA 12.1. Le test de Khi $2\left(\chi^{2}\right)$ a permis de comparer les effets de différents milieux de conditionnement, de différents âges de cals initiateurs de suspension cellulaire et de différents âges de suspensions cellulaires sur le taux de régénération de plantes. Pour chacune des caractéristiques considérées (taille des plantes, nombre de barres, longueur de la chandelle, longueur de l'épi et nombre de grains), le test $\mathrm{Z}$ a été utilisé pour comparer les valeurs moyennes des plantes régénérées à partir de suspensions cellulaires à celles des plantes issues de semis. Tous les tests statistiques ont été réalisés avec un risque d'erreur $\alpha=5 \%$.

\section{RÉSULTATS}

Effet de la taille de la fraction cellulaire et de l'âge de celle-ci sur le taux de régénération de plantes

Le nombre de plantes été régénérées par cal embryogène a varié entre deux et cinq (Figure 1d).

Le Tableau 1 présente l'effet de l'âge de la suspension cellulaire et de la taille des cals issus des fractions cellulaires sur le taux moyen de régénération des plantes. Pour chaque âge de suspension cellulaire, les cals issus des différentes fractions cellulaires ont des taux de régénération similaires. Le taux de régénération a été compris entre 93 et $97 \%$ pour la suspension cellulaire âgée d'un mois. Ce taux de régénération a diminué rapidement avec l'augmentation de l'âge de la suspension cellulaire embryogène. Ainsi, le taux de régénération a été de 82 à $85 \%$, de 46 à $51 \%$ et de 14 à $16 \%$ pour les suspensions cellulaires âgées respectivement de 2, 3 et 4 mois.
Évolution du taux de régénération de plantes en fonction du milieu de conditionnement

Le Tableau 2 montre que la régénération des plantes varie en fonction de l'âge de la suspension cellulaire. Il a été très élevé (environ 90\%) pour des suspensions cellulaires de 1 mois. Ce taux est passé à $80 \%$ pour des suspensions cellulaires de 2 mois, à $40 \%$ pour ceux de 3 mois pour atteindre environ $10 \%$ pour la suspension cellulaire de 4 mois et ce quelle que soit la fraction d'amas cellulaire et le milieu de conditionnement.

Les milieux de conditionnement et la dimension des amas cellulaires identifiés par les différentes fractions n'ont eu aucune influence sur le taux de régénération de plantes. Ainsi, pour le même âge, les trois milieux de conditionnement présentent des résultats similaires.

Effet de l'âge du cal initiateur de la suspension cellulaire et de l'âge de la suspension cellulaire sur le taux de régénération de plantes

Le Tableau 3 donne les moyennes des taux de régénération des plantes en fonction de l'âge du cal initiateur et de l'âge de la suspension cellulaire.

Pour chaque âge de suspension cellulaire, les taux de régénération ont été similaires quel que soit l'âge (2 à 12 mois) des cals initiateurs de la suspension cellulaire embryogène. Cependant, les taux de régénération de plantes ont été légèrement plus faibles lorsque la suspension cellulaire était issue de cals initiateurs âgés de 12 mois.

Pour chaque âge des cals initiateurs de la suspension cellulaire, le taux de régénération de plantes ont été compris entre 75 et $93 \%$ pendant les deux premiers mois de culture de la suspension cellulaire. Ce taux a chuté de moitié et pour se situer entre 39 et $46 \%$ au troisième mois de culture. Du troisième au quatrième mois de culture, le taux de régénération de plantes est devenu 
trois fois plus faible et s'est situé entre 11 et $15 \%$.

Évolution du taux de plantes normales en fonction de l'âge du cal initiateur de la suspension cellulaire et de l'âge de la suspension cellulaire

Le taux de plantes normales régénérées (Figure 1d) a varié en fonction de l'âge du cal initiateur de la suspension embryogène et de l'âge de la suspension embryogène (Tableau 4). Le taux de plantes normales diminue avec l'augmentation de l'âge des cals initiateurs et de l'âge des suspensions cellulaires. Les suspensions embryogènes obtenues à partir de cals de 2 à 3 mois ont permis une régénération de $70 \%$ de plantes normales. Ce taux est passé à $25 \%$ et $10 \%$ respectivement au bout de 3 et 4 mois de culture. Les suspensions embryogènes de 2 mois ont permis la régénération de $60 \%$ de plantes normales lorsqu'elles sont issues de cals initiateurs âgés de 2 à 3 mois. Ce taux est passé à $15 \%$ et 5\% respectivement pour des cals initiateurs âgés de 6 à 7 mois et de 12 mois.

Évolution du taux de plantes albinos en fonction de l'âge du cal initiateur de la suspension cellulaire et de l'âge la suspension cellulaire

Le taux de plantes albinos (Figure 1c) augmente avec l'augmentation de l'âge des cals initiateurs et de l'âge des suspensions cellulaires (Tableau 5). Les susepnsions embryogènes obtenues à partir de cals de 2 à 3 mois ont permis une régénération de $2 \%$ de plantes albinos. Ce taux est passé à $12 \%$ et $14 \%$ respectivement au bout de 3 et 4 mois de culture. Les suspensions embryogènes de 2 mois ont permis la régénération de $5 \%$ de plantes albinos lorsqu'ils sont issus de cals initiateurs âgés de 2 à 3 mois. Ce taux est passé à $20 \%$ et $45 \%$ respectivement pour des cals initiateurs âgés de 6 à 7 mois et de 12 mois. Pour ces deux derniers types de cals, les taux de plantes albinos ont atteint rapidement les 60 et $98 \%$ respectivement quand la suspension atteint 4 mois.

Comparaison de quelques caractéristiques de plantes régénérées et de plantes témoins

Les plantes régénérées à partir de suspensions embryogènes et les issues plantes de semis ont présenté les caractéristiques de croissance et de rendement consignées dans le Tableau 6. Pour ce qui concerne la longueur totale de la plante, elle a été de $200 \mathrm{~cm}$ pour les plantes issues de semis et $180 \mathrm{~cm}$ pour les plantes régénérées. Le nombre de barres a été de 18 pour les plants issus de semis et 20 pour les plantes régénérées. La longueur de l'épi a été de $9 \mathrm{~cm}$ pour les plantes issues de semis et $12 \mathrm{~cm}$ pour les plantes régénérées. Des fortes différences ont été observées seulement au niveau du nombre de grains par épi. (715 grains pour les plantes issues de semis et 412 grains pour les plantes régénérées).

Tableau 1 : Évolution du taux de régénération des plantes en fonction de l'âge de la suspension cellulaire et de la taille de la fraction cellulaire $(\%)$.

\begin{tabular}{ccccc}
\hline & \multicolumn{4}{c}{ Age de la suspension cellulaire (mois) } \\
\hline Fraction cellulaire & 1 & 2 & 3 & 4 \\
I & 93 & 82 & 46 & 16 \\
II & 96 & 85 & 48 & 14 \\
III & 97 & 84 & 51 & 16 \\
\hline
\end{tabular}

Fraction I = cals embryogènes de dimensions inférieures à $250 \mu \mathrm{m}$;

Fraction II = cals embryogènes de dimensions comprises entre $250 \mu \mathrm{m}$ et $630 \mu \mathrm{m}$;

Fraction III = cals embryogènes de dimensions supérieures à $630 \mu \mathrm{m}$. 
Tableau 2: Effet du milieu de conditionnement sur le taux de régénération de plantes fertiles (\%).

\begin{tabular}{lccccc}
\hline & & \multicolumn{4}{c}{ Age de la suspension cellulaire (mois) } \\
\hline Milieu de conditionnement & Fraction cellulaire & 1 & 2 & 3 & 4 \\
MS(1. 1. 2,5) & I & 93 & 82 & 46 & 16 \\
& II & 96 & 85 & 48 & 14 \\
& III & 97 & 84 & 51 & 16 \\
\hline MS(5) & I & 91 & 81 & 44 & 13 \\
& II & 93 & 82 & 49 & 16 \\
& III & 94 & 83 & 50 & 16 \\
\hline N6(1. 100. 25) & I & 94 & 82 & 48 & 14 \\
& II & 95 & 84 & 51 & 16 \\
& III & 95 & 84 & 52 & 17 \\
\hline
\end{tabular}

Tableau 3: Évolution du taux de régénération de plantes fertiles en fonction de l'âge du cal initiateur de la suspension cellulaire et de l'âge de la suspension cellulaire (\%).

\begin{tabular}{ccccc}
\hline & \multicolumn{4}{c}{ Age de la suspension cellulaire } \\
& \multicolumn{5}{c}{ (mois) } \\
\hline Age du cal initiateur de la suspension cellulaire (mois) & 1 & 2 & 3 & 4 \\
$2-3$ & 93 & 82 & 46 & 15 \\
$6-7$ & 92 & 84 & 45 & 13 \\
12 & 85 & 75 & 39 & 11 \\
\hline
\end{tabular}

Tableau 4: Évolution du taux de plantes normales en fonction de l'âge du cal initiateur de la suspension cellulaire et de l'âge de la suspension cellulaire (\%).

\begin{tabular}{|c|c|c|c|c|}
\hline \multicolumn{5}{|c|}{ Age de la suspension cellulaire (mois) } \\
\hline $\begin{array}{l}\text { Age du cal initiateur de } \\
\text { la suspension cellulaire } \\
\text { (mois) }\end{array}$ & 1 & 2 & 3 & 4 \\
\hline$(2-3)$ & 70 & 61 & 25 & 10 \\
\hline$(6-7)$ & 40 & 15 & 11 & 3 \\
\hline 12 & 10 & 5 & 0 & 0 \\
\hline
\end{tabular}

Tableau 5 : Évolution du taux d'albinisme en fonction de l'âge du cal initiateur de la suspension cellulaire et de l'âge de la suspension cellulaire (\%).

\begin{tabular}{cccccc}
\hline \multicolumn{5}{c}{ Age de la suspension cellulaire (mois) } \\
\hline $\begin{array}{c}\text { Age de l'amas de cals initiateur de la } \\
\text { suspension cellulaire (mois) }\end{array}$ & $\mathbf{1}$ & $\mathbf{2}$ & $\mathbf{3}$ & $\mathbf{4}$ \\
\hline $2-3$ & 2 & 5 & 12 & 14 \\
$6-7$ & 6 & 20 & 38 & 60 \\
12 & 28 & 45 & 80 & 98 \\
\hline
\end{tabular}


Tableau 6: Comparaison de quelques caractéristiques des plantes régénérées et des plantes issues de semis.

\begin{tabular}{lcc}
\hline Paramètres mesurés & Plantes témoins & plantes régénérées \\
\hline longueur totale $(\mathrm{cm})$ & $200+-4$ & $180+-3$ \\
nombre de barres & $18+-2$ & $20+-4$ \\
longueur de la chandelle $(\mathrm{cm})$ & $31+-2$ & $30+-1$ \\
longueur de l'epi $(\mathrm{cm})$ & $9+-2$ & $12+-1$ \\
nombre de grains par epi & $715+-195$ & $412+-278$ \\
\hline
\end{tabular}

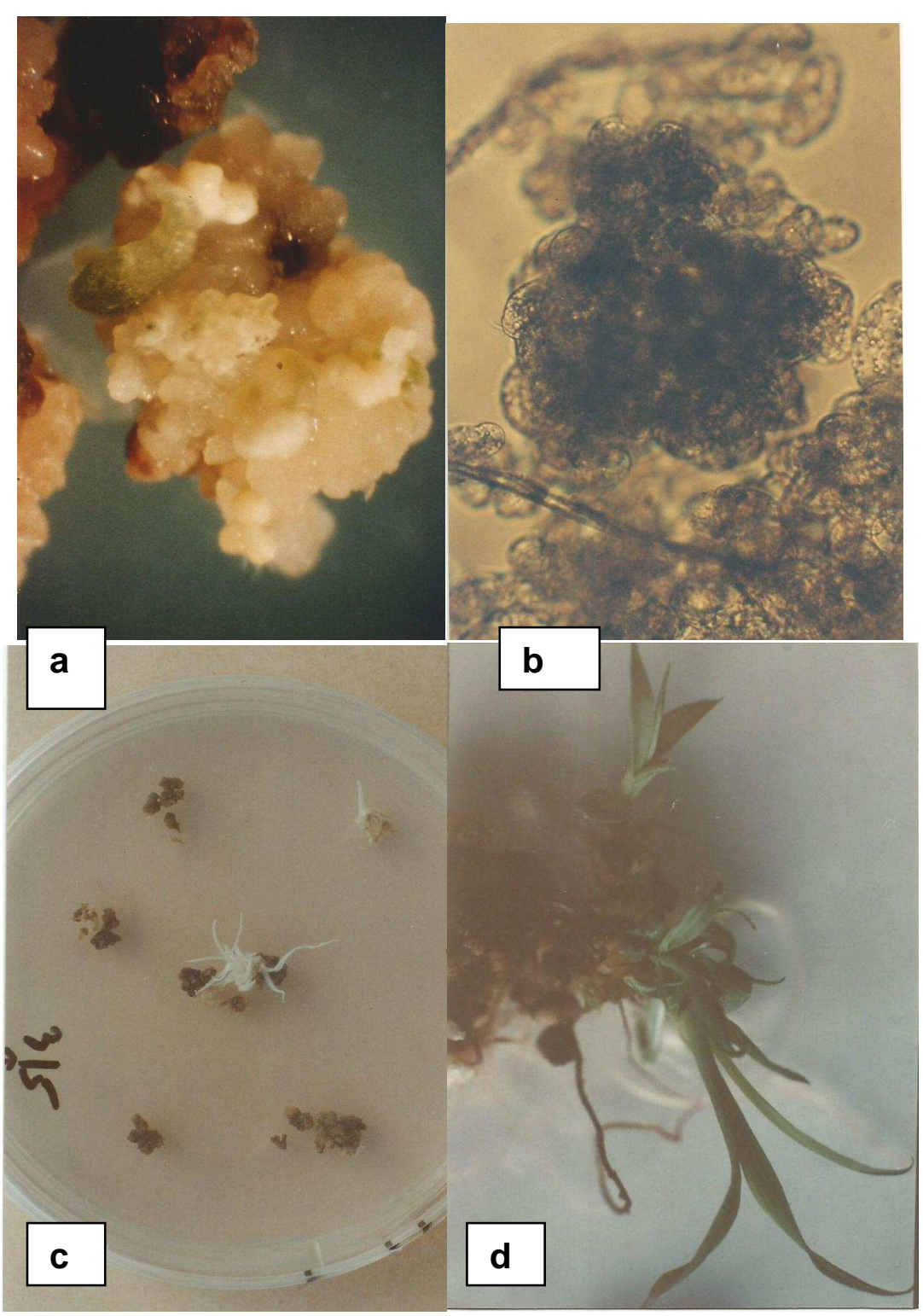

Figure 1: Régénération de plantes à partir de suspension cellulaire de mil (Pennisetum glaucum). a: cals embryogènes, b: Suspension cellulaire embryogène. $\mathbf{c}$ : plante albinos régénérée à partir de suspension cellulaire embryogène. $\mathbf{d}$ : jeunes plantes régénérées à partir de suspension cellulaire embryogène. 


\section{DISCUSSION}

La régénération de plantes est réalisée avec des suspensions cellulaires embryogènes issus de cals. Le maintien des cals, pendant une semaine, sur milieux gélosés avant transfert sur milieu de régénération, permet de donner un aspect sec et compact aux cals qui sortent du milieu liquide. Cet aspect compact peut être renforcé par l'utilisation du charbon (Lambé et al., 1999; Sharma et al., 2005). Nos résultats indiquent que Les fractions cellulaires I (cals embryogènes de dimensions inférieures à $250 \mu \mathrm{m}$ ), II (cals embryogènes de dimensions comprises entre $250 \mu \mathrm{m}$ et 630 $\mu \mathrm{m}$ ) et III (cals embryogènes de dimensions supérieures à $630 \mu \mathrm{m}$ ) ne montrent pas une différence pour ce qui est de leurs capacités à régénérer des plantes. Ce résultat constitue une donnée cognitive importante militant en faveur de l'utilisation de la fraction cellulaire I. Le choix de la fraction I tient au fait que cette fraction est la mieux indiquée pour les extractions de protoplastes et de transformations génétiques chez le mil (Tiécoura et al., 2001).

Dans la présente étude, nous avons rarement obtenu plus de 5 plants régénérées par cal embryogène issu de milieu de culture liquide. Tiécoura et al. (2003) ont par contre observé, en moyenne, une dizaine de plantes régénérées par cal embryogène solide de Pennisetum glaucum. Ces résultats suggèrent que les cals embryogènes solides de Pennisetum glaucum ont de plus fortes capacités de régénération de plantes que les cals embryogène issus de milieu de culture liquide.

Chez Pennisetum americanum, El Mtili (1990) a montré que la capacité des cals embryogènes à régénérer des plantes diminue à mesure que l'âge de la suspension cellulaire embryogène augmente. Le taux de régénération de plantes est par ailleurs nul sur des cals embryogènes de plus de 5 mois. Des plantes ont été régénérées à partir de cals embryogènes de seulement la moitié des génotypes étudiés et les taux de régénération ont varié de 5 à $30 \%$. Nos résultats sont conformes à ceux de El Mtili (1990) en ce qui concerne la diminution du taux de régénération de plantes à mesure que la suspension cellulaire vieillit. Cette diminution du taux de régénération s'explique par le fait que, dans le milieu liquide, les cals embryogènes sont friables et retrouvent difficilement leur aspect compact et sec quand ils sont transférés sur le milieu solide en vue de la régénération de plantes. Ces cals embryogènes perdent donc leur capacité de régénération lorsque la suspension cellulaire est âgée en moyenne de trois mois alors que Tiécoura et al. (2003) ont régénéré des plantes de $P$. americanum à partir de cals solides âgés de 10 à 12 mois.

Les taux de régénérations que nous avons obtenus sur des cals embryogènes âgés de 1 et 2 mois varient entre 75 et $93 \%$ et sont nettement supérieurs au meilleur taux de régénération (30\%) obtenu par El Mtili (1990). Ces données militent donc en faveur de l'utilisation de cals embryogènes issus d'explants d'apex caulinaire pour la régénération de plantes de $P$. americanum.

La plupart des cals embryogènes de Graminées ayant régénéré des plantes, proviennent de suspensions cellulaires obtenues à partir d'explants d'embryons matures ou immatures et d'inflorescences âgés de 3 à 6 semaines. La régénération de plantes n'a été faite qu'avec des suspensions cellulaires âgées de 2 mois au plus (Oldach et al., 2001 ; Sharma et al., 2005). Au-delà de deux mois, toutes les plantes régénérées sont albinos (Gamborg et al., 1970; Lu et al., 1981). Cependant, nos résultats montrent qu'il est possible de régénérer des plantes vertes avec des suspensions cellulaires âgées de 2 à 4 mois, à condition que l'âge de l'amas de cals ne soit pas plus de 5 mois.

Les plantes régénérées présentent les mêmes caractéristiques que celles issus de semis pour certains paramètres. Les différences observées au niveau des autres paramètres pourraient s'expliquer par le fait que le transfert de matériel des conditions de laboratoire au milieu naturel demande un 
certain délai d'adaptation. Cela a été mis en évidence par Yoboué et al. (2013) sur des plants issus de la régénération d'embryons zygotiques de cocotier et transférées dans les conditions naturelles.

\section{Conclusion}

Les résultats de ces travaux présentent les conditions optimales de régénération de plantes fertiles à partir de suspensions cellulaires issues cals embryogènes d'apex de Pennisetum glaucum. Au terme de cette étude, il apparait que :

-La régénération de plantes fertiles à partir de ces suspensions cellulaires embryogènes est possible.

- L'âge des cals embryogènes et celle de la suspension cellulaire influent sur la régénération de plantes fertiles. Une meilleure régénération requiert des cals et des suspensions cellulaires d'au plus 3 mois.

- Les milieux de culture ainsi que la taille des amas de cals utilisés n'ont pas d'influence sur le taux de régénération des plantes. Cependant, la fraction I constitue le matériel idéal, l'objectif final étant l'utilisation de ce matériel pour la transformation génétique.

- Les plantes régénérées présentent les mêmes caractéristiques que les plantes issues de semis. Cependant, elles ont besoin d'un certain délai d'adaptation dans les conditions naturelles.

Les meilleures conditions d'établissement des suspensions cellulaires et de régénération de plantes fertiles étant obtenues, il serait intéressant d'étudier l'usage de ce matériel pour la transformation génétique.

\section{REFERENCES}

Arockiasamy S, Ignacimuthu SS. 2007. Regeneration of transgenic plants from two indica rice (Oriza sativa L.) cultivars using shoot apex explant. Plant Cell Reports, 26(10): 1745-1753.

Arockiasamy S, Ignacimuthu SS. 2007. Regeneration of transgenic plants from two indica rice (Oriza sativa L.) cultivars using shoot apex explant. Plant Cell Reports, 26(10): 1745-1753.

Devi P, Zhong H, Sticklen MB. 2000. In vitro morphogenesis of pearl millet (Pennisetum glaucum L. R. Br.): efficient production of multiple shoots et inflorescences from shoot apices. Plant Cell Reports, 19(6): 546-550.

El Mtili N. 1990. Embryogenèse somatique et culture de cellules et de protoplastes chez le mil (Pennisetum americanum L.). Thèse de Doctorat. Université d'Orsay (France), p. 137.

Gnanaprasam S, Vasil IK. 1992. Ultrastructural changes in suspension culture cells of Panicum maximum during cryopreservation. Plant Cell Rep, 11: 169 - 174.

Haliloglu K. 2006. Efficient regeneration system from weat leaf base segments. Biol. Plant, 50: 326 - 330.

Jha P, Yadav CB, Anjalah V, Bhat V. 2009. In vitro plant regeneration through somatic embryogenesis et direct shoot organogenesis in Pennisetum glaucum (L.) R. Br. In Vitro Cell Dev. Biol. Plant, 45: 145-154.

Kamo KK, Hodges TK. 1986. Establishment and characterization of long-term embryogenic maize callus et cell suspension cultures. Plant Science, 45: 111-117.

Karp A, Wu QS, Steele SH, Jones MGK. 1987. Chromosome variation in dividing protoplasts et cell suspension of wheat. Theor. Appl. Genetics, 74: 140-146.

Koffi Y, N'Nan O, Konan JL, Malaurie B, Engelmann F. 2013. Morphological and agronomical characteristics of coconut (Cocos nucifera) palms from in vitro culture zigotic embryo. In Vitro Cell Dev. Biol. Plant, 49(5): 599-604.

Lambé P, Dinant M, Matagne RF. 1995. Differential long-term expression et methylation of the hygromycin phosphotransferase (hph) et $\beta$ glucuronidase (GUS) genes in transgenic 
pearl millet (Pennisetum glaucum) callus. Plant Science, 108(1): 51-62.

Lambé P, Mutambel HSN, Deltour R, Dinant M. 1999. Somatic embryogenesis in pear millet (Pennisetum glaucum): Strategies to reduce genotype limitation and to maintain long-term totipotency. Plant cell Tissue Organ Culture, 55: 23-29.

Lambé P, Dinant M, Deltour R. 2000. Transgenic pearl millet (Pennisetum glaucum L. Br.). Biotech. in Agriculture et Forestry, 46: 84-108.

Lu C, Vasil IK. 1981. Somatic embryogenesis et plant regeneration from freely suspended cells et cell groups of Panicum maximum Jacq. Ann. Botany, 48: 543-548.

Mohana PA, Karutha PS, Ramesh M. 2011. Efficient in vitro plant regeneration through leaf base derived callus cultures of abiotic stress sensitive popular Asian Indica rice cultivar IR64 (Oriza sativa). Acta Biol. Hung. 62(4): 441 - 452.

Oldach KH, Morgenstern A, Rother S, Girgi M. 2001. Efficient in vitro plant regeneration from immature zygotic embryos of pearl millet (Pennisetum glaucum L. R. Br.) et Sorghum bicolor (L.) Moench. Plant Cell Reports, 20: 416-421.

Sharma VK, Hansch R, Mendel RR, Schulze J. 2005. Mature embryogenesis-based hight frequency somatic embryogenesis and plant regeneration from multiple cultivars of barley (Hordeum vulgare L.). J Ex Botany, 56(417): 1913-1922.

Shillito RD, Carswell GK, Johnson CM, Dimalo J, Harms CT. 1989.
Regeneration of fertile plants from protoplasts of elite inbred maize. Biotechnology, 7: 581-587.

Tiécoura K, Ledoux L, Dinant M. 2003. Expression transitoire du gène de la Bglucuronidase (Gus) dans les protoplastes de mil (Pennisetum glaucum (L) R) : étude comparative de deux promoteurs, CaMV35S et Emu. Sciences et Techniques, 25(2) : 87 - 97.

Tiécoura K, Ledoux L, Dinant M. 2003. Plant regeneration through tissue culture of pearl millet (Pennisetum glaucum (L.) R.). Agronomie Africaine, 15(3): 105 121.

Vasil IK. 1994. Molecular improvement of cereals. Plant Mol. Biology, 25: 925937.

Vasil V, Vasil IK. 1981. Somatic embryogenesis and plant regeneration from suspension cultures of Pearl millet (Pennisetum americanum). Ann. Botany, 47: 669-678.

Vasil V, Vasil IK. 1982. Caracterization of an embryogenic cell suspension culture derived from cultured inflorescences of Pennisetum amercanum (pearl millet, Gramineae). Amer. J. Botany, 69: 1441 1449.

Winfield M, Daavey MR. Karp A. 1993. A comparison of chromosome instability in cell suspensions of diploid, tetraploid and hexaploid wheats. Heredity. 70: 187-194.

Zimny J, Lörd H. 1986. Plant regeneration et initiation of cell suspensions from roottip derived callus of Oriza sativa L. (rice). Plant Cell Reports, 5: 89-92. 